\title{
STUDIES OF THE CARRIER STATE FOLLOWING INFECTION WITH GROUP A STREPTOCOCCI. I. EFFECT OF CLIMATE *
}

\author{
BY RICHARD M. KRAUSE, $\nmid$ CHARLES H. RAMMELKAMP, JR., FLOYD W. \\ DENNY, JR. AND LEWIS W. WANNAMAKER $¥$
}

(From the Streptococcal Disease Laboratory, Francis E. Warren Air Force Base, Wyo., and the Departments of Preventive Medicine and Medicine, Western Reserve University School of Medicine, Cleveland, Ohio)

(Submitted for publication September 5, 1961; accepted October 6, 1961)

The persistence of the group A streptococcus in the upper respiratory tract after pharyngitis has been termed the streptococcal carrier state. $\mathrm{Pa}$ tients with acute pharyngitis and individuals who continue to harbor the organism serve as the principal sources of the infectious agent in the spread to susceptible hosts (1). There is considerable variation in the number of streptococci found, however, and in the length of time that streptocoocci can be isolated from the throat after infection. In the presence of suppurative complications patients may discharge large numbers of organisms for many weeks. In the absence of such suppurative complications, only 40 to 70 per cent of patients continues to harbor the organism after 3 to 7 weeks in sufficient numbers for detection by a routine culture of the pharynx (2-4). Because the characteristics of the streptococcal carrier state have not been clearly defined, studies were undertaken of convalescent carriers after respiratory infections with epidemic strains of group A streptococci. As a part of this study the effect of climate on the carrier state was investigated, and the results form the basis for this report.

\section{METHODS}

Population studied. All airmen with respiratory symptoms entering the hospital between 11 and 27 March, 1951, at Warren Air Force Base at Cheyenne, Wyoming, received no specific antibacterial therapy. On admission a history and physical examination were recorded and a

* This investigation was conducted under the sponsorship of the Commission on Streptococcal and Staphylococcal Diseases and the Commission on Acute Respiratory Diseases, Armed Forces Epidemiological Board, and was supported by the Offices of the Surgeons General, Departments of the Army and the Air Force, Washington, D. C.

† Present address: Rockefeller Institute for Medical Research, New York, N. Y.

$\ddagger$ Career Investigator, American Heart Association. culture of the oropharynx was obtained. Those individuals who showed 10 or more colonies of $\beta$-hemolytic streptococci on the blood agar plate and whose illness was characteristic of a streptococcal infection were eligible for the study group. Of the patients meeting these criteria, 65 were available for study for at least 12 weeks. Selection of patients to be sent to a semitropical climate was limited to those individuals who had recently arrived and were awaiting assignment to the various schools at the air base. Twenty-nine patients met this qualification and were assigned to the group to be transferred to Florida; the remaining men were studied in Wyoming. The patients who remained in Wyoming for study had enrolled recently in the training schools. Thus, the patients who were transferred to Florida had been at Warren Air Force Base for a somewhat shorter time than those who remained at Warren. It should be emphasized, however, that both groups of men had been in the service several months for basic training prior to their assignment at Warren and were not new recruits.

Of the 36 patients remaining in Wyoming, 11 were dropped from the study. Six patients developed a streptococcal infection caused by a new streptococcal type; two developed acute rheumatic fever and received therapy with penicillin; two received antibiotics for a urinary tract infection and a perirectal abscess, respectively; one patient was transferred to another installation early in the study.

No patient assigned to Florida was dropped from the study; Patient 492 was transferred after 12 weeks of observation. Two definite cross infections occurred ( $\mathrm{Pa}$ tients 507,513 ) before transfer to Florida, but since they occurred in the first 2 weeks of illness and subsequent cultures failed to show the type causing the original infection, they are included in the analysis. Two patients developed mild symptoms of rheumatic fever but no antibacterial therapy was administered.

Routine procedures. Prior to 5 April when 29 patients were transferred by air transport ${ }^{1}$ to Tyndall Air Force Base at Panama City, Fla., cultures of the oropharynx and nose were taken at irregular intervals. Beginning on 8 April both nose and oropharyngeal cultures were

1 The authors express their sincere appreciation to General Edward J. Kendricks, MC, USAF, and his staff for making the necessary administrative arrangements for this study. 
obtained twice weekly from all patients. Oropharyngeal cultures were taken by rubbing both tonsils or tonsillar fossae twice and the posterior oropharynx at least three times. Nasal cultures were obtained by introducing one sterile swab into both nares for a distance of $3 \mathrm{~cm}$ followed by gentle rotation. Both the oropharyngeal and nasal swabs were immediately rubbed on sheep bloodagar plates. If either of the two cultures from the preceding period exhibited less than $10 \beta$-hemolytic colonies, a second culture was obtained and placed in Pike's selective medium (5). With few exceptions all cultures were taken by the authors, two of whom rotated between the study groups in Florida and Wyoming.

The inoculated blood agar plates were returned to the laboratory, and the inoculum was spread in a standard manner with a sterile bacteriological loop. Cuts were made in the agar plate with the loop in the region of heaviest inoculum to facilitate recognition of subsurface hemolysis. The blood-agar plates were incubated for 24 hours at $37^{\circ} \mathrm{C}$ and examined for $\beta$-hemolytic colonies, and the number of colonies on each plate was recorded. Swabs placed in Pike's medium were incubated for 24 hours and one loopful was inoculated on a sheep bloodagar plate as described above. After incubation the plate was examined for $\beta$-hemolytic colonies, but the number present was not recorded. From each plate a single representative colony was subcultured on a sheep blood-agar plate for confirmation. In Florida, a subculture from the confirmation plate was made on a blood-agar slant and sent to Wyoming by air mail for serological grouping and typing. In Wyoming, serological identification was performed on organisms obtained from the confirmation plate. Thus, on each day that cultures were taken, a maximum of four colonies was identified completely from the cultures of each individual.

One colony obtained from the oropharyngeal culture each week was rapidly frozen and stored at $-20^{\circ}$ or
TABLE I

Comparability of the two study groups

\begin{tabular}{lcc}
\hline & \multicolumn{2}{c}{ Location of patients } \\
\cline { 2 - 3 } & $\begin{array}{c}\text { Wyoming } \\
(25 \text { men })\end{array}$ & $\begin{array}{c}\text { Florida } \\
(29 \text { men })\end{array}$ \\
\hline Average days since onset of & & \\
illness to 5 April & 17 & 15 \\
Per cent with: & & \\
Tonsillectomy & 16 & 55 \\
Total leukocyte count & & \\
over 10,000 & 83 & 89 \\
Antistreptolysin response & 71 & 85 \\
Exudative tonsillitis or & 96 & 97 \\
pharyngitis & & \\
Number infected by: & & \\
Type 1 & 5 & 4 \\
5 & 6 & 3 \\
6 & 1 & 4 \\
14 & 0 & 17 \\
41 & &
\end{tabular}

$-70^{\circ} \mathrm{C}$ and served as a source of organisms for mouse inoculation.

The media employed were supplied by the laboratories in Wyoming and the sheep blood was obtained from a common source. Tryptose blood-agar base (Difco) with 6 per cent sterile sheep blood was employed for the original plates. Confirmation plates utilized beef heart infusion broth with added agar and sheep blood. Standard procedures were employed for serological identification of the isolated streptococcal strains (6).

A serum specimen was taken on admission to the hospital, every 2 weeks for 6 weeks, and every month until the study was terminated. Antistreptolysin $O$ titers were performed by standard techniques ( 7 ).

TABLE II

Weather conditions at Panama City, Florida and Cheyenne, Wyoming during period of study*

\begin{tabular}{llcccc}
\hline \hline & & \multicolumn{3}{c}{ Month } \\
\cline { 4 - 6 } & Location & April & May & June & July \\
\hline Mean temperature $\left({ }^{\circ} F\right)$ & Panama City & 65.9 & 74.2 & 80.4 & 83.0 \\
& Cheyenne & 44.2 & 50.9 & 51.3 & 67.1 \\
Mean max. temper- & Panama City & 72.6 & 81.5 & 85.7 & 88.8 \\
ature $\left({ }^{\circ} F\right)$ & Cheyenne & 48.7 & 62.9 & 66.4 & 80.5 \\
Mean min. temper- & Panama City & 58.8 & 66.7 & 74.5 & 77.0 \\
ature $\left({ }^{\circ} F\right)$ & Cheyenne & 25.9 & 39.4 & 42.8 & 53.1 \\
Mean relative humidity & Panama City & 75.6 & 70.6 & 77.6 & 75.9 \\
Precipitation $($ inches $)$ & Cheyenne & 65.5 & 62.4 & 68.9 & 59.2 \\
Mean wind speed & Panama City & 4.01 & 2.72 & 7.16 & 10.41 \\
$(m p h)$ & Cheyenne & 1.94 & 2.77 & 1.76 & 2.74 \\
& Panama City & 8.6 & 8.1 & 6.7 & 5.1 \\
\end{tabular}

* Data supplied by Air Weather Service, Data Control Unit, New Orleans, La. 


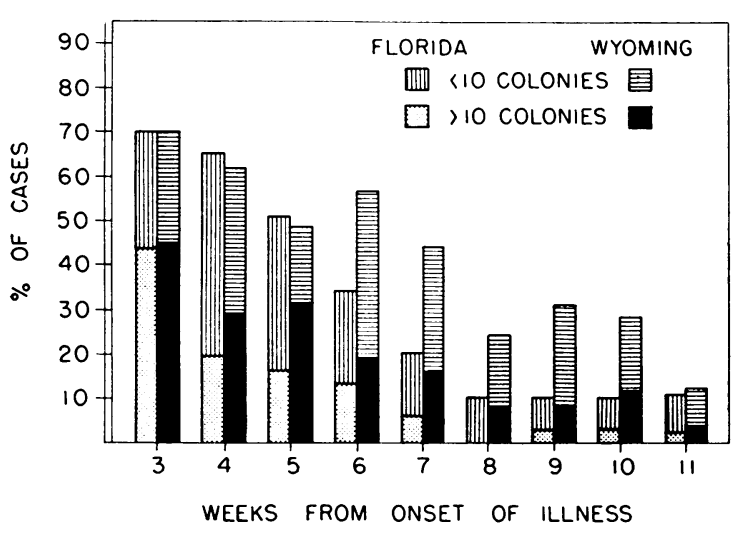

Fig. 1. Persistence of group A streptococci in NASAL CULTURES AFTER INFECTION.

Records of all symptoms and abnormal physical signs were maintained throughout the study. Special studies, including roentgenograms of the sinuses, electrocardiograms, and sedimentation rates were obtained as indicated.

\section{RESULTS}

The characteristics of the carrier state were studied in 54 young adult males who developed symptoms of a streptococcal respiratory infection between 11 and 25 March, 1951, at Cheyenne, Wyoming. The study assessed the possible effects of changes in climate on the carrier state by dividing the patients into two groups and following the convalescence of one group in Wyoming and that of the other after transfer to Florida. On 5 April one group of 29 airmen was transported to Panama City, Florida, and the second group of 25 men remained in Wyoming. The two groups appeared to be relatively comparable. except that 55 per cent of those transferred to Florida had had tonsillectomy, whereas only 16 per cent of the those remaining in Wyoming had had this operation (Table I). At the time the men were transferred to Florida, the average interval since the onset of illness in the two groups was 2 weeks. The clinical, bacteriological, and serological evidence established the streptococcal etiology of these respiratory illnesses. The majority of the infections was due to the three epidemic strainstypes 14,1 , and 5 (4).

The differences in climate during convalescence in Florida and Wyoming are presented in Table II. The men in Wyoming were at an altitude of 6,000 feet, and for 6 to 8 weeks the temperature was low and snow frequently covered the ground.
The men were housed in multiple two-floor army barracks. They attended regular classes and were in contact with many individuals harboring group A streptococci, since epidemic conditions continued through June.

The group in Florida was housed in a single one-floor hospital ward situated a few hundred yards from the Gulf of Mexico. Initially the weather was mild and later quite warm so that the windows in the ward were usually open. The men were assigned duties outdoors on the hospital grounds, and were, in general, indoors only when sleeping.

Figures 1 and 2 summarize the quantitation of oropharyngeal and nasal cultures, respectively. For this analysis the maximum number of streptococci isolated on the blood-agar plates for each week of observation is presented. At the third week, when nasal cultures were instituted, 70 per cent of both groups in Wyoming and Florida showed positive nose cultures, and approximately 45 per cent of the cultures in each group exhibited more than 10 colonies. Both groups showed a decline in positive cultures during the study, so that by week 11 only 10 per cent was positive, and from these only a few organisms were isolated. At week 4, however, a difference in the percentage of cultures showing more than $10 \mathrm{col}-$ onies became apparent, the rate being higher in the Wyoming than in the Florida group. Furthermore, during the sixth week the total rate of all positive nasal cultures decreased more rapidly in the Florida subjects. Additional analysis indicated that this difference in the nasal carrier rate between the two groups was not due to the fact

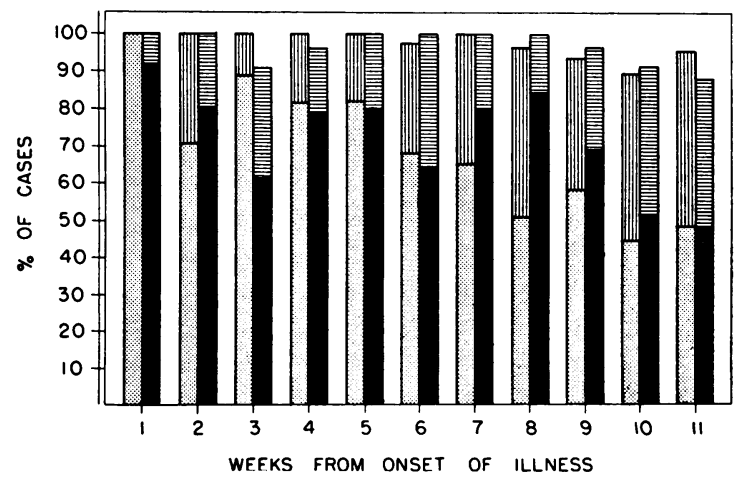

Fig. 2. Persistence of group A StReptococci in throat cultures after infection. See Figure 1 for key to symbols. 


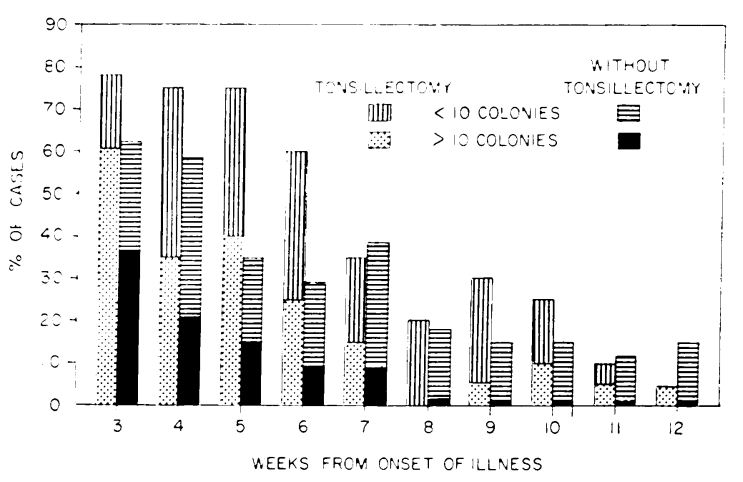

Fig. 3. Persistence of group A streptococci in XASAl CUltures after INFECTION IN ALL Patients OF BOTH GROUPS WITH AND WITHOUT TONSILLECTOMY.

that a greater proportion of those patients in Florida had had tonsillectomy. A comparison was made of the persistence of group A streptococci in the nasal cultures for all patients in the study with and without tonsillectomy. The results as tabulated in Figure 3 indicate that the nasal carrier rate was somewhat higher for a longer period for those patients who had had tonsillectomy than for those who had not. For instance, during week 6, 60 per cent of all patients in both groups with tonsillectomy were nasal carriers, while only 28 per cent of the nontonsillectomy patients continued to harbor the organism in the nose. Because there were so few patients in Wyoming with tonsillectomy it was impossible to compare them with a similar group in Florida. An additional analysis was therefore made of the persistance of the nasal carrier in only those patients without tonsillectomy in Florida and Wyoming. As can be seen in Fig-

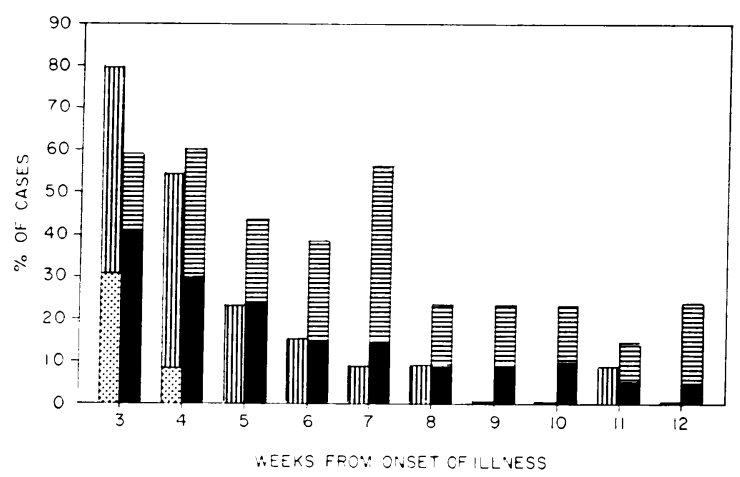

Fig. 4. Persistence of group A streptococci in NASAl Clltures after INFection in those patients without tonsillectomy. See Figure 1 for key to symbols.

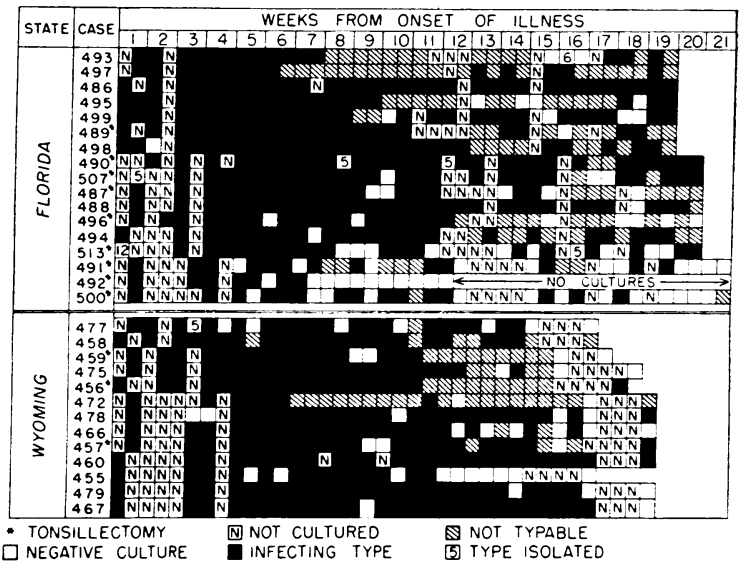

Fig. 5. Biweekly clltures of patients with type 14 STREPTOCOCCAL INFECTIONS.

ure 4 , beginning with week 4 and continuing throughout the period of observation, the rate of positive nasal cultures was higher for the patients in Wyoming than for the Florida group. These data strengthen the view that the nasal carrier is more likely to persist in a north temperate climate than in a semitropical region.

In contrast to the relatively rapid disappearance of streptococci from the anterior nares. cultures of the oropharynx were usually positive during the first 11 weeks of observation (Figure 2). Quantitative cultures revealed no significant difference between the two groups. By week 11 cultures from over half of the patients exhibited less than 10 colonies. As seen in Figures 5 and 6 , the study was extended past 11 weeks, but because of technical difficulties the 28 subjects remaining

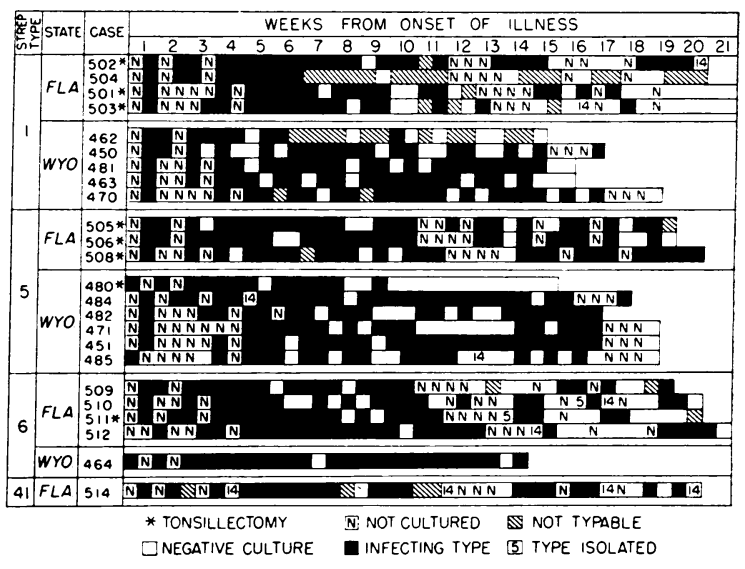

Fig. 6. Biweekly cultures of patients with types $1,5,6$, AND 41 STREPTOCOCCAL INFECTIONS. 


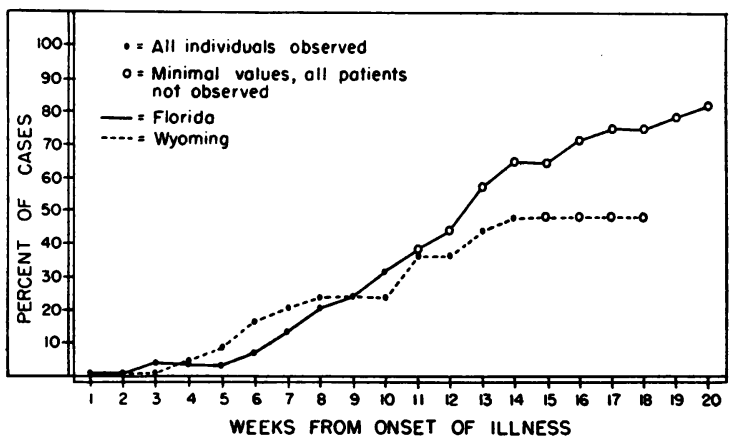

Fig. 7. Cumulative Frequency of NONTYPable group A STREPTOCOCCI.

in Florida were not cultured regularly for a period of 2 weeks, and after week 16 the Wyoming group was not cultured systematically.

The results of the semiweekly cultures of the individual subjects listed according to the infecting type are recorded on Figures 5 and 6 . That the carrier state continued for many weeks, although individual cultures were occasionally negative, is emphasized by the fact that all of the 28 subjects in Florida between weeks 16 and 19 harbored group A streptococci.

Of considerable interest was the appearance of nontypable variants of the infecting type as the carrier state progressed. Figure 7 summarizes the development of nontypable variants in the two groups. Beginning at about week 3, non-M protein-producing organisms made their appearance, and by week 11 , cultures from 40 per cent of the patients showed such organisms. There appeared to be a difference in the rate of development of such variants during subsequent weeks with the suggestion that the cumulative frequency of nontypable group A streptococci in Florida was greater than that in Wyoming. Because the numbers of cultures obtained in Wyoming and Florida between weeks 11 and 21 are not comparable, it is difficult to interpret the difference between the two groups. Infections with types 1 and 5 were followed by the appearance of nontypable variants more frequently in the Florida than in the Wyoming group (Figure 6). Thus, 6 of 7 subjects in Florida, and 2 of 11 in Wyoming showed these strains. Sixteen selected strains of the nontypable variants were serially passed in the peritoneal cavities of mice, and in each instance the nontypable strains reverted to the original infecting type.

\section{DISCUSSION}

A particular feature of streptococcal diseases is that they are more prevalent in colder climates than in tropical or semitropical areas $(8,9)$. Although the opportunities for effective intimate contact between the carrier and the susceptible host may be greater in population groups confined to buildings because of inclement weather, it is possible that climate might exert an effect on the host which would indirectly modify the metabolism of the bacteria residing in the upper respiratory tract. It is possible that the respiratory tract of the host in colder areas might maintain the infectious properties of the streptococcus for more prolonged periods. Likewise, the spread of infection might be enhanced in northern areas because the climatic conditions render the upper respiratory passages more susceptible to infections. The present study was originally designed to test the hypothesis that climate alters the mucous membranes of the upper respiratory tract in such a manner that the streptococcal carrier in colder climates presents a more suitable environment for the maintenance of the organisms than does the carrier who lives in a semitropical area. If such were the case, this might be evidenced by a prolonged carrier state and by a more prolific growth of organisms in the respiratory tract of those patients who reside in north temperate zones.

Using the duration of the carrier state as the primary criterion for evaluating the effect of climate, it is clear that there was no demonstrable influence of climatic conditions on the length of time that the oropharyngeal cultures remained positive for hemolytic streptococci. These studies do emphasize, however, that almost all individuals who acquire an infection with an epidemic strain of streptococci continue to harbor the organism for at least 3 or 4 months. This is in contrast to the studies of Kuttner and Krumwiede (10), who showed that 61 of 118 patients harbored the infecting organisms for only 3 months or less; most of the remaining 57 patients continued to harbor the organisms for many months, and in a few instances for a year and a half. In other'studies from our laboratory (4), in which the protocol for follow-up cultures was much less rigorous than that employed here, the infecting organism was isolated from only 53 per cent of the patients 
during the fourth or fifth week of convalescence. The fact that the streptococci remain in small numbers for extended periods has important implications concerning the interpretation of culture surveys of various population groups. The isolation of a few colonies of group A streptococci may indicate infection many months previously. If the organism is not typable, it is unlikely that infection was acquired in the recent past. ${ }^{2}$

The present study emphasizes the persistence of group A streptococci in the oropharynx after infection with epidemic strains. The persistence of organisms after infection by strains not associated with streptococcal epidemics has not been thoroughly studied, but there is some information to suggest that after infection with nonepidemic strains the carrier state is of shorter duration (11).

Although cultures of the oropharynx showed no difference in the carrier state between the groups of men in Florida and Wyoming, cultures of the anterior nares showed that organisms disappeared more rapidly from the men in Florida. These differences in the nasal cultures could not be attributed to clinically evident acute or chronic sinusitis. Furthermore, additional analysis indicated that removal of the tonsils, and presumably the adenoids, was not responsible for the fewer positive nasal cultures in the Florida carriers. Indeed, the data suggested that the nasal carrier state may persist longer in those patients with a tonsillectomy, thus confirming the observations of Holmes and Williams (11). Gordon (2), Hamburger, Green and Hamburger (12), and Wannamaker (1) have presented evidence indicating that the nasal carrier is especially likely to serve as a source of infection. It is interesting, therefore, that in the northern climate of Wyoming, where streptococcal sore throats are more common, the dangerous nasal carrier state persisted longer than in Florida. The data, however, are insufficient to do more than suggest that this effect is due to climatic conditions.

There was a slight difference in the rate of appearance of nontypable variants of group A streptococci in the cultures from the respiratory tracts of the Wyoming and Florida patients, but this did not become evident until the fourth month

\footnotetext{
2 In some instances the identification of an organism as a nontypable variant means that the proper typing antisera were not employed.
}

after the onset of infection. If these trends reflect the effect of environment on the biological activities of the streptococcus, the organism might be considered to be maintained in a more virulent or infective state in the colder climate. Further studies would be required to establish such a relationship.

Studies on the treatment of acute streptococcal infection indicate that the carrier state may play a role in the pathogenesis of acute rheumatic fever. Treatment with antibiotics which does not eliminate the infecting organism fails to decrease the attack rate of rheumatic fever (13). More recently it has been shown the rheumatic fever may be prevented after a streptococcal sore throat if penicillin is given as late as 9 days after the onset of the streptococcal illness (14). These data suggest that it is the elimination of the streptococci from the throat and not the early treatment of the acute pharyngitis, per se, that prevents the occurrence of rheumatic fever. In studies of rheumatic fever attack rates after streptococcal infections there is some evidence indicating that epidemics are associated with constant rates of about 3 per cent (15), whereas after endemic infections the rates may be much lower $(11,16)$. These differences may be due to variability in the persistence of the carrier state. Additional studies on the carrier state are needed to elucidate the mechanisms for the maintenance of the organism in the population and the relationship of the carrier state to rheumatic fever.

\section{SUMMARY}

The carrier state was studied in 54 young adult males after streptococcal sore throat. The patients were divided into two groups: one group remained in Wyoming for extended convalescence, and the other was transferred to Florida. Almost all patients continued to harbor group A hemolytic streptococci in the oropharynx for 16 to 19 weeks, at which time the study was terminated. In many of the patients a nontypable variant of the infecting type of streptococcus developed during the period of observation. There was no evidence that the change in climate had any effect on the length of the carrier state or the numbers of organisms isolated from the pharynx, although nontypable variants appeared slightly earlier and streptococci dis- 
appeared from the anterior nares sooner in the group transferred to a warmer climate.

\section{ACKNOWLEDGMENT}

The authors wish to express their appreciation to Col. Wayne Pittman, MC, USAF, Surgeon, Tyndall Air Force Base, and to the technical staff of the Streptococcal Disease Laboratory.

\section{REFERENCES}

1. Wannamaker, L. W. The epidemiology of streptococcal infections in Streptococcal Infections, $M$. McCarty, Ed. New York, Columbia Univ. Press, 1954, p. 157.

2. Gordon, J. E. Hemolytic streptococci in the upper respiratory tract of scarlet fever convalescents. J. prev. Med. (Baltimore) 1927, 1, 289.

3. Commission on Acute Respiratory Diseases. A study of a food-borne epidemic of tonsillitis and pharyngitis due to B-hemolytic streptococci, type 5. Bull. Johns Hopk. Hosp. 1945, 77, 143.

4. Catanzaro, F. J., Brock, L., Chamovitz, R., Perry, W. D., Siegel, A. C., Stetson, C. A., Rammelkamp, C. H., Jr., Houser, H. B., Stolzer, B. L., Wannamaker, L. W., and Hahn, E. O. Effect of oxytetracycline therapy of streptococcal sore throat on the incidence of acute rheumatic fever. Ann. intern. Med. 1955, 42, 345.

5. Pike, R. M. Enrichment broth for isolating hemolytic streptococci from throat swabs. Proc. Soc. exp. Biol. (N. Y.). 1944, 57, 186.

6. Swift, H. F., Wilson, A. T., and Lancefield, R. C. Typing group A hemolytic streptococci by $M$ precipitin reactions in capillary pipettes. J. exp. Med. 1943, 78, 127.

7. Hodge, B. E., and Swift, H. F. Varying hemolytic and constant combining capacity of streptolysins: Influences on testing for antistreptolysins. J. exp. Med. 1933, 58, 277.
8. Holbrook, W. P. The Army Air Forces rheumatic fever control program. J. Amer. med. Ass. 1944, 126, 84.

9. Paul, J. R. The Epidemiology of Rheumatic Fever. New York, American Heart Ass., 1957, pp. 100107.

10. Kuttner, A. G., and Krumwiede, E. Observations on the epidemiology of streptococcal pharyngitis and the relation of streptococcal carriers to the occurrence of outbreaks. J. clin. Invest. 1944, 23, 139.

11. Holmes, M. C., and Williams, R. E. O. Streptococcal infections among children in a residential home. I. Introduction and definitions: The incidence of infection. J. Hyg. (Lond.) 1958, 56, 43.

12. Hamburger, M., Jr., Green, M. R., and Hamburger, V. G. The problem of the "dangerous carrier" of hemolytic streptococci. II. Spread of infection by individuals with strongly positive nose cultures who expelled large numbers of hemolytic streptococci. J. infect. Dis. 1945, 77, 96.

13. Catanzaro, F. J., Rammelkamp, C. H., Jr., and Chamovitz, R. Prevention of rheumatic fever by treatment of streptococcal infections. II. Factors responsible for failures. New Engl. J. Med. 1958, 259, 51.

14. Catanzaro, F. J., Stetson, C. A., Morris, A. J., Chamovitz, R., Rammelkamp, C. H., Jr., Stolzer, B. L., and Perry, W. D. The role of the streptococcus in the pathogenesis of rheumatic fever. Amer. J. Med. 1954, 17, 749.

15. Rammelkamp, C. H., Jr., Wannamaker, L. W., and Denny, F. W. The epidemiology and prevention of rheumatic fever. Bull. N. Y. Acad. Med. 1952, 28, 321.

16. Stetson, C. A., Jr. The relation of antibody response to rheumatic fever in Streptococcal Infections, M. McCarty, Ed. New York, Columbia Univ. Press, 1954, p. 208. 